\title{
Efficacy of Epidural Neuroplasty Versus Transforaminal Epidural Steroid Injection for the Radiating Pain Caused by a Herniated Lumbar Disc
}

\author{
Hae Jong Kim, $\mathrm{MD}^{1}$, Byeong Cheol Rim, MD², Jeong-Wook Lim, MD², Noh Kyoung Park, MD ${ }^{1}$, \\ Tae-Wook Kang, $\mathrm{MD}^{1}$, Min Kyun Sohn, $\mathrm{MD}^{3}$, Jaewon Beom, $\mathrm{MD}^{3}$, Sangkuk Kang, $\mathrm{MD}^{1}$ \\ Departments of ${ }^{1}$ Physical Medicine and Rehabilitation and ${ }^{2}$ Neurosurgery, Sun General Hospital, Daejeon; \\ ${ }^{3}$ Department of Physical Medicine and Rehabilitation, Chungnam National University Hospital, Daejeon, Korea
}

\begin{abstract}
Objective To compare the treatment effects of epidural neuroplasty (NP) and transforaminal epidural steroid injection (TFESI) for the radiating pain caused by herniated lumbar disc.

Methods Thirty-two patients diagnosed with herniated lumbar disc through magnetic resonance imaging or computed tomography were included in this study. Fourteen patients received an epidural NP and eighteen patients had a TFESI. The visual analogue scale (VAS) and functional rating index (FRI) were measured before the treatment, and at 2 weeks, 4 weeks and 8 weeks after the treatment.

Results In the epidural NP group, the mean values of the VAS before the treatment, and at 2 weeks, 4 weeks and 8 weeks after the treatment were $7.00 \pm 1.52,4.29 \pm 1.20,2.64 \pm 0.93,1.43 \pm 0.51$ and those of FRI were $23.57 \pm 3.84$, $16.50 \pm 3.48,11.43 \pm 2.44,7.00 \pm 2.15$. In the TFESI group, the mean values of the VAS before the treatment, and at 2 weeks, 4 weeks and 8 weeks after the treatment were $7.22 \pm 2.05,4.28 \pm 1.67,2.56 \pm 1.04,1.33 \pm 0.49$ and those of FRI were $22.00 \pm 6.64,16.22 \pm 5.07,11.56 \pm 4.18,8.06 \pm 1.89$. During the follow-up period, the values of VAS and FRI within each group were significantly reduced $(\mathrm{p}<0.05)$ after the treatment. But there were no significant differences between the two groups statistically.

Conclusion Epidural NP and TFESI are equally effective treatments for the reduction of radiating pain and for improvement of function in patients with a herniated lumbar disc. We recommend that TFESI should be primarily applied to patients who need interventional spine treatment, because it is easier and more cost-effective than epidural NP.
\end{abstract}

Keywords Epidural neuroplasty, Transforaminal epidural steroid injection, Radiating pain, Herniated lumbar disc 


\section{INTRODUCTION}

It is known that the induction of radiating pain caused by a herniated lumbar disc is not simply due to mechanical compression [1] but is also due to the release of different kinds of inflammatory mediators including phospholipase A2 and prostaglandin E2 from the herniated nucleus pulposus [2]. For the treatment of patients with lower limb radiating pain caused by radiculopathy due to a herniated lumbar disc, there are both surgical and conservative approaches. It has been reported that in the case of conservative treatment, there was not much difference in terms of treatment efficacy compared to the surgical method after a lapse of more than a year $[3,4]$. Especially, the 'transforaminal epidural steroid injection (TFESI)' is a popular conservative treatment due to its effectiveness in reducing the inflammatory reaction caused by the herniated lumber disc. Among the three epidural steroid injection methods, TFESI, interlaminar epidural steroid injection, and caudal epidural steroid injection, the TFESI is the most effective method because TFESI enables the injection of a high dose of steroid directly into the ventral epidural space where the lesion is located $[5,6]$.

Epidural adhesion most commonly occurs from postoperative hemorrhage into the epidural space and hemorrhage during the subsequent recovery period, or from fibrocyte deposition and inflammatory reaction from intervertebral disc extrusion into the epidural space caused by disc herniation [7]. According to the study by Racz and colleagues [8-10], 'epidural neuroplasty (NP)' or 'epidural adhesiolysis' is more effective treatment for patients who have epidural adhesions caused by a herniated lumbar disc because a catheter is placed directly at the adhesion site for the performance of the adhesiolysis before the epidural injection is given, so that the injected solution can more easily spread through the adhesion. Recently, epidural NP has been frequently carried out for the same reason, but it is more costly and it has not yet been proven to be better than TFESI. Thereby, this prospective study was done to observe the amount of pain reduction and functional improvement and compare them between the patient groups with lumbar disc herniation that were given the different therapies of epidural NP and TFESI.

\section{MATERIALS AND METHODS}

\section{Subjects}

The subjects of this study were 32 enrolled outpatients aged between 20 to 60 years, who had visited the department of neurosurgery or rehabilitation medicine at the spine center between the January to December 2012 for their lower limb radiating pain, diagnosed as radiculopathy caused by lumbar disc herniation, based on the patient interview, physical examination, and lumbar computed tomography (CT) or magnetic resonance imaging (MRI). The patients had not shown any symptomatic improvement while receiving conservative treatment, such as medication or physiotherapy for at least more than 4 weeks. Fourteen ( 6 male, 8 female) of the patients received epidural NP from the department of neurosurgery, and eighteen ( 8 male, 10 female) of them received TFESI from the department of rehabilitation medicine. On the other hand, based on medical imaging techniques, those patients showing hypertrophy of the posterior longitudinal ligament and ligamentum flavum, and lumbar spinal stenosis caused by a vertebral body and osteophytes were excluded. In addition, the patients who had steroid injections or surgery in the lumbar area, or who were severe diabetics with uncontrolled blood glucose, or had myelopathies or cauda equina lesions were also excluded from the subject groups.

\section{Method}

The epidural NP was performed by an experienced neurosurgeon, and the TFESI was performed by an experienced physiatrist. The procedure was conducted under minimal radiation exposure, and both the conductor and assistant put on lead aprons, goggles, and thyroid protectors during the procedures.

\section{Lumbosacral epidural neuroplasty}

In the epidural NP, the modified Racz protocol described by Manchikanti et al. $[11,12]$ was used. In the Carm fluoroscopic room, venous entry was secured prior to the main procedure, then the blood pressure, pulse, and pulse oximetry were monitored during the procedure. First, the patient was placed in the prone position on the C-arm table, and from the fluoroscopic anterioposterior view, a 16-gauge R.K. needle (Epimed International, Farmers Branch, TX, USA) was inserted into the 
sacral hiatus, then into the epidural space. Contrast medium was injected to determine the region of the filling defect (Fig. 1A), and the catheter was moved to execute mechanical adhesiolysis. Following this, a second injection of contrast medium was performed to outline the epidural space and nerve root (Fig. 1B). When the target nerve was enhanced, a mixture of Pucaine $0.5 \% 4 \mathrm{~mL}$ (bupivacaine), dexamethasone $5 \mathrm{mg}$, and Hyalase Dessau 1500 IU (hyaluronidase) was slowly injected. In cases that showed no filling defect, the catheter was introduced into the place of the lesion viewed on the lumbar CT or MRI and an injection with the same medication was performed.

\section{Lumbosacral transforaminal epidural steroid injection}

The protocol adopted for the TFESI was described by Fenton and Czervionke [13].

1) The L4 and L5 nerve root TFESI: The patient was placed in the prone position on the $\mathrm{C}$-arm table and the injection site was disinfected. The C-arm was rotated obliquely towards the ipsilateral side of the target nerve root. When the "Scotty dog appearance" was verified, a spinal needle $22-\mathrm{G} \times 3^{1 / 2}$ inches (TaeChang Industrial co., Gongju, Korea) was inserted just below the pedicle which corresponds to Scotty dog's eye. After slowly proceeding with the spinal needle injection until it provoked radiating pain, the contrast medium was put in to make sure that the nerve root could be clearly visualized (Fig. 2A), and then a total of a $2 \mathrm{~mL}$ mixture of $2 \%$ lidocaine $0.5 \mathrm{~mL}$, triamcinolone acetonide $20 \mathrm{mg}$, and normal saline 1 mL was injected.

2) The S1 nerve root TFESI: After disinfecting the injection site of the patient lying prone on the C-arm table, cephalic tilting of the patient was done to verify the radiolucent $\mathrm{S} 1$ foramen which is the overlap of the ventral and dorsal sacral foramina. The spinal needle was slowly moved towards the radiolucent S1
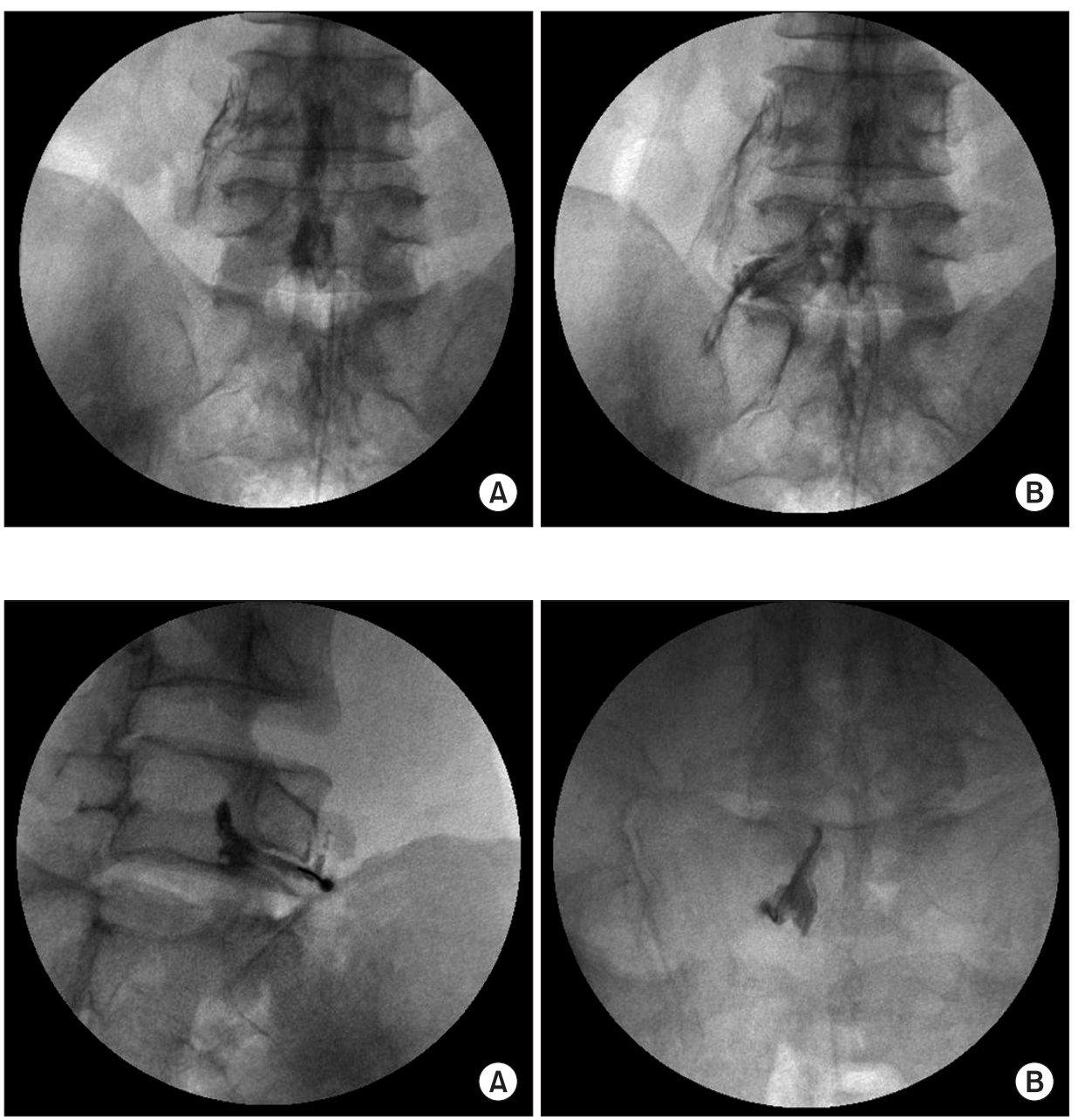

Fig. 2. Transforaminal epidural steroid injection (TFESI) (A) Fluoroscopic oblique view after administering the right L5 TFESI with contrast medium, showing the right L5 nerve root. (B) Fluoroscopic anterioposterior view after administering the left S1 TFESI with contrast medium, showing the S1 nerve root. 
foramen until radiating pain was provoked. When the nerve root was demonstrated by the contrast medium injection (Fig. 2B), the same medication was given.

\section{Criteria of clinical improvement}

In order to evaluate the degree of clinical improvement in the patients who had undergone lumbosacral epidural NP and TFESI, the medical resident at the department of rehabilitation medicine assessed the 'visual analogue scale (VAS)' of the radiating pain and the 'functional rate index (FRI)' [14-16] before the procedure and at 2, 4, and 8 weeks after the procedure. Only those patients in the TFESI group who had no symptomatic improvement or showed only a minor VAS improvement of less than $20 \%$ at the 2 -week point after the procedure were given an additional injection.

\section{Statistical analysis}

To perform the statistical analysis, a non-parametric test was implemented by SPSS for Window ver. 18.0 (SPSS Inc., Chicago, IL, USA). The degree of improvement as scored by the VAS and FRI was analyzed by the Wilcoxon signed rank test and compared between the two groups, and the effectiveness of the treatment at each time interval was analyzed by the Mann-Whitney U-test and compared between the two groups. The confidence interval (CI) considered was $95 \%(95 \% \mathrm{CI})$ and a $\mathrm{p}<0.05$ was considered significant.

\section{RESULTS}

\section{Patient group characteristics}

The average age of the epidural NP group was $45.29 \pm$ 9.83 years and the TFESI group was $48.11 \pm 11.69$ years. The average duration of symptoms in the epidural NP group was $19.07 \pm 10.75$ weeks, and in the TFESI group, it was $19.00 \pm 14.52$ weeks. Referring to the age and duration of symptoms, there was no significant statistical difference between the two groups. The number of each target nerve root site in the epidural NP group was two L4s, fifteen L5s, and five S1s, and in the TFESI group, there were one L4, nineteen L5s, and three S1s. Eight patients in the epidural NP and five in the TFESI group had more than two ipsilateral dermatomes or bilateral pain radiation, and they received the same procedure on both the nerve roots. Also, after two weeks of tracking observation, only those four subjects in the TFESI group showing slight or no pain relief received an extra injection (Table 1).

\section{Efficacy of the treatments compared by VAS}

Before the treatment, the VAS was $7.00 \pm 1.52$ in the epidural NP group and 7.22 \pm 2.05 in the TFESI group. At 2, 4, and 8 weeks later, the VAS became $4.29 \pm 1.20,2.64 \pm 0.93$, and $1.43 \pm 0.51$ in the epidural NP group and $4.28 \pm 1.67$, $2.56 \pm 1.04$, and $1.33 \pm 0.49$ in the TFESI group, respectively. The pain within each group was significantly reduced $(\mathrm{p}<0.05)$ after the treatments, and there were no significant statistical differences between the two groups (Fig. 3).

\section{Efficacy of the treatments compared by FRI}

Before the treatment, FRI was $23.57 \pm 3.84$ in the epidural NP group and 22.00 \pm 6.64 in the TFESI group. At 2, 4, and 8 weeks later, FRI became $16.50 \pm 3.48,11.43 \pm 2.44$, and 7.00 \pm 2.15 in the epidural NP group and 16.22 \pm 5.07 , $11.56 \pm 4.18$, and $8.06 \pm 1.89$ in the TFESI group, respectively. The functions within each group were significantly improved $(\mathrm{p}<0.05)$ after the treatments, but there were no significant statistical differences between the two groups (Fig. 4).

Table 1. Demographic characteristics of subjects of both epidural NP and TFESI

\begin{tabular}{lcc}
\hline & $\begin{array}{c}\text { Epidural NP } \\
(\mathbf{n}=\mathbf{1 4})\end{array}$ & $\begin{array}{c}\text { TFESI } \\
(\mathbf{n}=\mathbf{1 8})\end{array}$ \\
\hline Gender (male:female) & $6: 8$ & $8: 10$ \\
\hline Age (yr) & $45.29 \pm 9.83$ & $48.11 \pm 11.69$ \\
\hline Duration of symptom (wk) & $19.07 \pm 10.75$ & $19.00 \pm 14.52$ \\
\hline Leg pain distribution & & \\
$\quad$ 1 Level & 6 & 13 \\
\hline 2 Levels (ipsilesional:bilateral) & $6: 2$ & $3: 2$ \\
Target root & & \\
$\quad$ L4 & 2 & 1 \\
\hline L5 & 15 & 19 \\
\hline S1 & 5 & 3 \\
Repeated procedure person & 0 & 4 \\
Hypertension & 3 & 4 \\
Diabetes mellitus & 1 & 1 \\
\hline
\end{tabular}

Values are presented as mean \pm standard deviation or number.

Epidural NP, epidural neuroplasty; TFESI, transforaminal epidural steroid injection. 


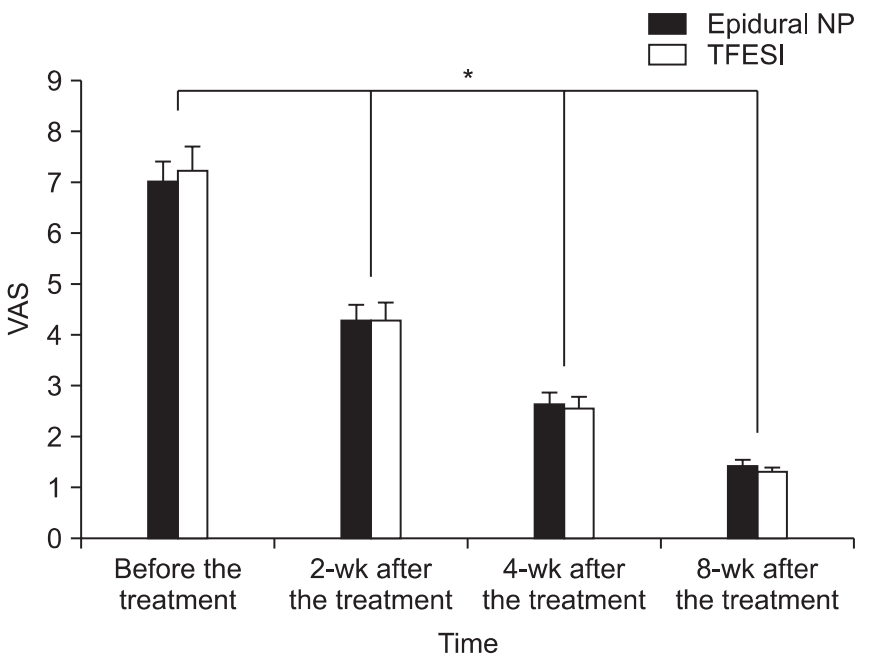

Fig. 3. The changes of the visual analogue scale (VAS) over the 8-week follow-up. Epidural NP: epidural neuroplasty, TFESI: transforaminal epidural steroid injection. ${ }^{*} \mathrm{p}<0.05$ comparison between before the treatment and post-treatment by Wilcoxon signed rank test in both the epidural NP and TEFSI groups.

\section{DISCUSSION}

After 1989 when epidural NP was first described by Racz and Holubec [8], there have been reports about its effectiveness in treating chronic low back pain and lower limb radiating pain $[11,12,17,18]$. Mechanical adhesiolysis by the catheter introduced into the scar tissue at the epidural space in the course of epidural NP enables the injected medication to permeate better through the adhesion site. Epidural adhesion and fibrosis usually follows after lumbar spinal surgery and is caused by the infiltration of fibrous connective tissue into the post-surgical hematoma [19]. There are three steps in the pathophysiology of epidural adhesions: first, the inflamed pia mater and arachnoid mater cause congestion and edema of the cauda equina followed by fibrocyte and collagen deposition along the nerve root and nerve membrane. Second, the nerve roots become adhered to by the fibrocytes and collagen, and adhesion between the nerve roots and the cerebrospinal fluid membrane takes place. In the third step, ischemia and contraction caused by the collagenous membrane formed around the nerve root alters the neurophysiology to the state of functional loss and excitement [20]. Epidural adhesion can also take place in cases of myelography, spinal anesthesia, and multiple spinal puncture [21]. Also epidural NP is performed on

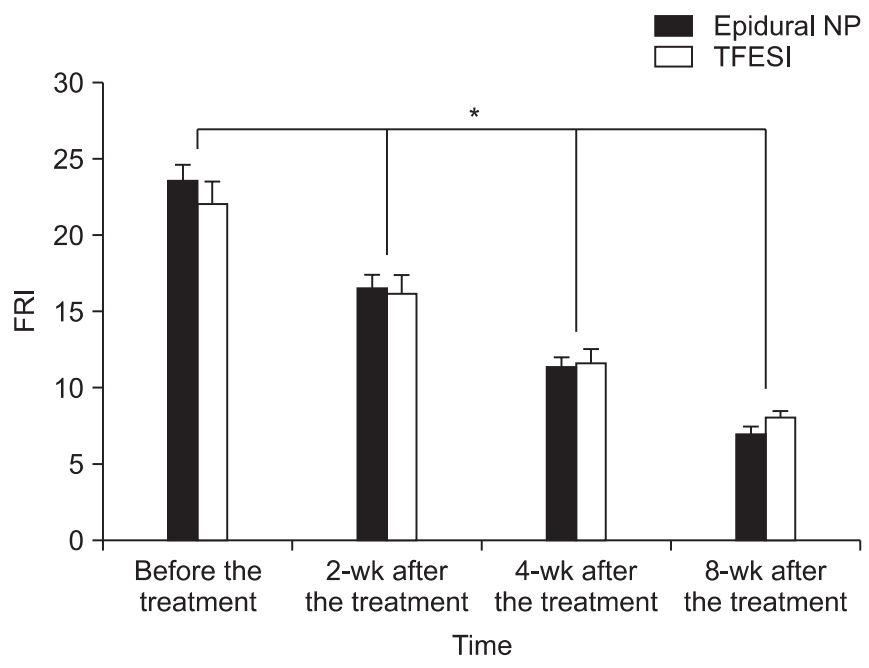

Fig. 4. The changes of the functional rate index (FRI) for the 8-week follow-up period. Epidural NP: epidural neuroplasty, TFESI: transforaminal epidural steroid injection. ${ }^{*} \mathrm{p}<0.05$ comparison between before the treatment and post-treatment by Wilcoxon signed rank test in both the epidural NP and TEFSI groups.

in the case of a herniated lumbar disc, because of lateral or dorsal leaked nucleus pulposus through ruptured annulus fibrosus induce fibrocytes infiltration, inflammatory response and adhesion [7]. In addition to steroids and local anesthetics, hyaluronidase, isotonic saline, and hypertonic saline are included among the epidural NP injection solutions. Hyaluronidase is a spreading factor that improves the diffusion of the medication through the scar tissue inside the epidural space, and there are reports describing the increased efficacy of the treatment in patients who had received a hyaluronidase injection prior to the local anesthetic compared to patients who had not [9]. Plus, there has been comparative research showing no distinction between the group that had been injected with hyaluronidase and isotonic saline and the group that had received hyaluronidase and hypertonic saline [19]. Therefore, in this research, the mixed solution of a steroid, local anesthetic, hyaluronidase and isotonic saline was injected after the catheter using mechanical adhesiolysis for treating lower limb radiating pain from radiculopathy caused by a herniated lumbar disc. From the eighth consecutive week of tracking observation, it was statistically possible to detect a reduction in the pain and functional improvement. According to the research about the complications from epidural injections [22], intravascular entry, local hematoma, transient 
nerve root irritation, dural puncture, profuse bleeding, bruising, post-lumbar puncture headache and several other complications developed among patients who had received an epidural NP (albeit rarely), but there were no such complications appearing in the 14 patients in this research study.

Epidural steroid injections have been widely used as a conservative method for treating lumbar and lower limb radiating pain. It is known that TFESI produces a better result than inter-laminar or caudal epidural steroid injection because of the direct steroid injection into the selected inflamed epiradicular nerve root sheath $[5,6]$. Also, the absence of adverse effects from high-dose steroids and the capability of direct injection into a lesion make it a lot easier to find the area of the lesion or to decide on the site of surgery [23,24]. A combined injection solution of a steroid, local anesthetic, and isotonic saline used for TFESI reduces the pain-provoking inflammatory reaction that is not only induced by simple compression of the vertebral disc but also by inflammatory mediators including phospholipase A2 and prostaglandin E2 [25,26]. In addition, a local anesthetic is used together with a steroid for its effect in blocking the nociceptor signaling pathway [27] and reducing the nerve root tension that causes radiating pain [28]. In this research as well, patients with lower limb radiating pain caused by herniated lumbar disc radiculopathy statistically showed similar pain reduction and functional improvement for eight consecutive weeks after the TFESI treatment. According to a study about the complications of epidural injections [22], intravascular entry of the spinal needle, transient nerve root hypersensitivity, local hematoma, facet joint entry of the spinal needle, bruising, profuse bleeding, facial flushing, vasovagal reaction, disc entry of the spinal needle and several other complications (rarely) developed among patients receiving TFESI, but there were no such complications for the 18 patients in this research. From this study, there was no clear evidence to support the claim that epidural NP is better than TFESI in terms of pain reduction and functional improvement. This is presumably because the adhesions and fibrosis caused by the herniated lumbar disc were less severe than the postsurgical adhesions and fibrosis, so that as a result, the effect of the mechanical adhesiolysis by epidural NP could not be so prominent. Also, it could be due to the fact that in both groups, the medication was given selectively at the ventral epidural space where the spinal disc herniation most commonly occurs, causing mechanical pressure on the nerve root or inflammation of the posterior longitudinal ligament [29]. A cost comparison of the two procedures in the United States showed that TFESI was about $52 \%$ of the price of epidural NP [30]. In South Korea, however, there was a big gap between the two procedures: the cost of TFESI was only $10 \%$ that of epidural NP, due to differences in medical insurance coverage in the two countries.

The constraints in this research were the short tracking observation period of eight weeks for making comparisons, the different medical operators in the two groups, and the low statistical validity due to the small number of subjects in each group (14 and 18, respectively). Moreover, the steroid that was used in the epidural NP was 5 $\mathrm{mg}$ dexamethasone but $20 \mathrm{mg}$ triamcinolone was used in the TFESI, and thus a lesser dose of the steroid was used in the epidural NP group. Despite the unequal steroid doses, there would not have been many pharmacological differences considering the fact that the ratio of the potency of dexamethasone relative to triamcinolone is 0.75:4 mg [31]. We believe a long-term tracking observation and clinical study on a larger scale for more precise comparison of the treatment effect in those two groups is required.

According to this research, there were recognizable changes in pain reduction and functional improvement in the daily life until eight weeks after the epidural NP but statistically its effect was not so prominent compared to the TFESI treatment for lower limb radiating pain caused by a herniated lumbar disc radiculopathy. Therefore, we recommend that TFESI be primarily applied to patients who need interventional spine treatment, because it is easier and more cost-effective than epidural NP.

\section{CONFLICT OF INTEREST}

No potential conflict of interest relevant to this article was reported.

\section{REFERENCES}

1. Boden SD, Davis DO, Dina TS, Patronas NJ, Wiesel SW. Abnormal magnetic-resonance scans of the lumbar spine in asymptomatic subjects: a prospective investigation. J Bone Joint Surg Am 1990;72:403-8. 
2. Saal JS, Franson RC, Dobrow R, Saal JA, White AH, Goldthwaite N. High levels of inflammatory phospholipase A2 activity in lumbar disc herniations. Spine (Phila Pa 1976) 1990;15:674-8.

3. Weinstein JN, Lurie JD, Tosteson TD, Skinner JS, Hanscom B, Tosteson AN, et al. Surgical vs nonoperative treatment for lumbar disk herniation: the Spine Patient Outcomes Research Trial (SPORT) observational cohort. JAMA 2006;296:2451-9.

4. Weinstein JN, Tosteson TD, Lurie JD, Tosteson AN, Hanscom B, Skinner JS, et al. Surgical vs nonoperative treatment for lumbar disk herniation: the Spine Patient Outcomes Research Trial (SPORT): a randomized trial. JAMA 2006;296:2441-50.

5. Lutz GE, Vad VB, Wisneski RJ. Fluoroscopic transforaminal lumbar epidural steroids: an outcome study. Arch Phys Med Rehabil 1998;79:1362-6.

6. Vad VB, Bhat AL, Lutz GE, Cammisa F. Transforaminal epidural steroid injections in lumbosacral radiculopathy: a prospective randomized study. Spine (Phila Pa 1976) 2002;27:11-6.

7. McCarron RF, Wimpee MW, Hudkins PG, Laros GS. The inflammatory effect of nucleus pulposus: a possible element in the pathogenesis of low-back pain. Spine (Phila Pa 1976) 1987;12:760-4.

8. Racz GB, Holubec JT. Lysis of adhesion in the epidural space. In: Racz GB, editor. Techniques of neurolysis. Boston: Kluwer Academic Publisher; 1989. p. 57-72.

9. Racz GB, Heavner JE, Diede JH. Lysis of epidural adhesions utilizing the epidural approach. In: Waldman $\mathrm{SD}$, Winnie AP, editors. Interventional pain management. Philadelphia: Saunders; 1996. p. 339-51.

10. Racz GB, Heavner JE, Trescot A. Percutaneous lysis of epidural adhesions: evidence for safety and efficacy. Pain Pract 2008;8:277-86.

11. Manchikanti L, Rivera JJ, Pampati V, Damron KS, McManus CD, Brandon DE, et al. One day lumbar epidural adhesiolysis and hypertonic saline neurolysis in treatment of chronic low back pain: a randomized, double-blind trial. Pain Physician 2004;7:177-86.

12. Manchikanti L, Pampati V, Fellows B, Rivera J, Beyer CD, Damron KS. Role of one day epidural adhesiolysis in management of chronic low back pain: a randomized clinical trial. Pain Physician 2001;4:153-66.

13. Fenton DS, Czervionke LF. Image-guided spine intervention. Philadelphia: Saunders; 2003.
14. Chansirinukor W, Maher CG, Latimer J, Hush J. Comparison of the functional rating index and the 18-item Roland-Morris Disability Questionnaire: responsiveness and reliability. Spine (Phila Pa 1976) 2005;30:141-5.

15. Grotle M, Brox JI, Vollestad NK. Functional status and disability questionnaires: what do they assess? A systematic review of back-specific outcome questionnaires. Spine (Phila Pa 1976) 2005;30:130-40.

16. Feise RJ, Michael Menke J. Functional rating index: a new valid and reliable instrument to measure the magnitude of clinical change in spinal conditions. Spine (Phila Pa 1976) 2001;26:78-86.

17. Veihelmann A, Devens C, Trouillier H, Birkenmaier C, Gerdesmeyer L, Refior HJ. Epidural neuroplasty versus physiotherapy to relieve pain in patients with sciatica: a prospective randomized blinded clinical trial. J Orthop Sci 2006;11:365-9.

18. Heavner JE, Racz GB, Raj P. Percutaneous epidural neuroplasty: prospective evaluation of $0.9 \% \mathrm{NaCl}$ versus $10 \% \mathrm{NaCl}$ with or without hyaluronidase. Reg Anesth Pain Med 1999;24:202-7.

19. LaRocca H, Macnab I. The laminectomy membrane. Studies in its evolution, characteristics, effects and prophylaxis in dogs. J Bone Joint Surg Br 1974;56:545-50.

20. Burton CV. Lumbosacral arachnoiditis. Spine (Phila Pa 1976) 1978;3:24-30.

21. Benner B, Ehni G. Spinal arachnoiditis: the postoperative variety in particular. Spine (Phila Pa 1976) 1978;3:40-4.

22. Manchikanti L, Malla Y, Wargo BW, Cash KA, Pampati V, Fellows B. A prospective evaluation of complications of 10,000 fluoroscopically directed epidural injections. Pain Physician 2012;15:131-40.

23. Woodward JL, Herring SA, Windsor RE, Dreyer SJ, Lester JP, Lagattuta FP. Epidural procedures in spine pain management. In: Lennard TA, editor. Physiatric procedures in clinical practice. Philadelphia: Hanley \& Belfus; 1995. p. 260-91.

24. Woodward JL, Weinstein SM. Epidural injections for the diagnosis and management of axial and radicular pain syndromes. Phys Med Rehabil Clin N Am 1995;6:691-714.

25. Lee HM, Weinstein JN, Meller ST, Hayashi N, Spratt KF, Gebhart GF. The role of steroids and their effects on phospholipase A2: an animal model of radiculopathy. Spine (Phila Pa 1976) 1998;23:1191-6. 
26. Kawakami M, Matsumoto T, Tamaki T. Roles of thromboxane A2 and leukotriene B4 in radicular pain induced by herniated nucleus pulposus. J Orthop Res 2001;19:472-7.

27. Kirkaldy-Willis WH, Bernand TN. A comprehensive outline of treatment. In: Kirkaldy-Willis WH, Bernand TN, editors. Managing low back pain. New York: Churchill Livingstone; 1983. p. 147-60.

28. Evans W. Intrasacral epidural injection in the treatment of sciatica. Lancet 1930;216:1225-9.
29. Winkel D, Aufdemkampe G, Matthijs O, Meijer OG, Phelps V. Diagnosis and treatment of the spine. Gaithersburg: Aspen Publishers; 1996.

30. Manchikanti L. Transforaminal lumbar epidural steroid injections. Pain Physician 2000;3:374-98.

31. Hardman JG, Limbird LE, Molonoff PB, Ruddon RW, Gilman AG. Goodman \& Gilman's the pharmacological basis of therapeutics. 9th ed. New York: McGrawHill; 1996. 